\title{
Coupled first-order transitions and unconventional superfluidity in a Fermi-Bose mixture
}

\author{
K. Sheshadri $\oplus^{1, *}$ and A. Chainani $\oplus^{2, \dagger}$ \\ 1226, Bagalur, Bangalore North, Karnataka State, 562149, India \\ ${ }^{2}$ Condensed Matter Physics Group, National Synchrotron Radiation Research Center, Hsinchu 30076, Taiwan
}

(Received 8 December 2019; revised manuscript received 18 May 2020; accepted 20 May 2020; published 5 June 2020)

\begin{abstract}
A model of a mixture of spinless fermions and spin-zero hardcore bosons, with filling fractions $\rho_{F}$ and $\rho_{B}$, respectively, on a two-dimensional square lattice with composite hopping $t$ is presented. In this model, hopping swaps the locations of a fermion and a boson at nearest-neighbor sites. When $\rho_{F}+\rho_{B}=1$, the fermion hopping amplitude $\phi$ and boson superfluid amplitude $\psi$ are calculated in the ground state within a mean-field approximation. The Fermi sector is insulating $(\phi=0)$ and the Bose sector is normal $(\psi=0)$ for $0 \leqslant \rho_{F}<\rho_{c}$. The model has coupled first-order transitions at $\rho_{F}=\rho_{c} \simeq 0.3$, where both $\phi$ and $\psi$ are discontinuous. The Fermi sector is metallic $(\phi>0)$ and the Bose sector is superfluid $(\psi>0)$ for $\rho_{c}<\rho_{F}<1$. At $\rho_{F}=1 / 2$, fermion density of states $\rho$ has a van Hove singularity, the bulk modulus $\kappa$ displays a cusplike singularity, the system has a density wave (DW) order, and $\phi$ and $\psi$ are maximum. At $\rho_{F}=\rho_{\kappa} \simeq 0.81, \kappa$ vanishes, becoming negative for $\rho_{\kappa}<\rho_{F}<1$. The evolution of fermion effective mass, Fermi band dispersions, and Fermi surfaces as a function of $\rho_{F}$ is highlighted. The obtained value for the ratio of the estimated superfluid $T_{c}$ to the fermion bandwidth, of approximately 0.12 , agrees closely with reported values of the ratio for a variety of unconventional superfluids and superconductors with $T_{c}$ values spread over nine orders of magnitude.
\end{abstract}

DOI: 10.1103/PhysRevResearch.2.023291

\section{INTRODUCTION}

Fermi-Bose mixtures (FBMs) constitute an unusual phase of matter, the earliest examples of which are the mixed phase of type-II superconductors and ${ }^{3} \mathrm{He}-{ }^{4} \mathrm{He}$ mixtures [1]. In the past two decades, fascinating experiments on FBMs of ultracold atoms have revealed unique properties and given a major thrust to their study [2-4]. While superfluidity was observed very early in either the Fermi or Bose sector of an FBM, the coexistence of superfluidity in both sectors was reported very recently [5].

Theoretical and experimental studies of FBMs have developed significantly over the years, particularly in terms of a crossover between the limiting cases of the BCS picture of superconductivity and the BEC picture of superfluidity [6,7]. The BCS-BEC crossover was predicted to occur for excitons in semiconductors [8], quarks [9], and, interestingly, it was first realized in ultracold fermionic atoms with $s$-wave interactions [10]. The growing number of experimental results includes the Feshbach resonance across the BCS-BEC crossover [11], formation of a Feshbach molecule in an FBM [12], and the role of three body physics in describing an FBM [13] to name a few. More recently, experimental results of some

\footnotetext{
*kshesh@gmail.com

†chainania@gmail.com
}

Published by the American Physical Society under the terms of the Creative Commons Attribution 4.0 International license. Further distribution of this work must maintain attribution to the author(s) and the published article's title, journal citation, and DOI. iron-based superconductors and the relevance of their electronic structure and properties [14-17] have also been discussed in relation to theoretical results of the BCS-BEC crossover $[6,18,19]$. While it is well known that interactions between fermions mediated by phonons are at the root of the BCS theory of superconductivity, several studies have also considered their importance in FBMs of ultracold atoms [20-23]. The BCS theory was preceded by the so-called boson-fermion (BF) model [24], which discusses itinerant fermions interacting with localized bosons composed of bound pairs of fermions of opposite spins. The BF model was later applied to study electrons interacting with local lattice deformations [25] and high temperature superconductivity [26-29]. More recently, it was also used for describing resonance superfluids in the BCS-BEC crossover regime [30] as well as a temperature driven BCS-BEC crossover in an FBM [31]. The interplay of bosonic and fermionic degrees of freedom is therefore of great importance in several physical systems.

Based on the measured values of the critical temperature $T_{c}$ and estimated bandwidths $W$ (or the Fermi temperature $T_{F}=E_{F} / \mathrm{k}_{B}$, where $E_{F}$ is the Fermi energy) for a variety of unconventional superfluids and superconductors, including ultracold FBMs in the BEC-BCS crossover regime, high$T_{c}$ cuprates, twisted bilayer graphene, sulfur hydride $\mathrm{H}_{3} \mathrm{~S}$, lanthanum hydride $\mathrm{LaH}_{10}$, etc., it is known that the ratio $T_{c} / W$ (or $T_{c} / T_{F}$ ) is in the range of $\sim 0.03-0.2[5,32-44]$. It was recognized very early by Uemura that systems with a relatively high $T_{c} / T_{F}$ are very different [45] from conventional BCS-type superconductors [46] which exhibit a very low $T_{c} / T_{F} \sim 10^{-5}-10^{-4}$. In a very recent review, Kagan and Bianconi have discussed models of FBMs and strong coupling ap- 
proaches for understanding unconventional superconductors which include high- $T_{c}$ cuprates, iron-based superconductors, metal oxide superconducting interfaces, the recently discovered superconducting hydrides under pressure, etc. [47]. In particular, the authors emphasized the importance of a Lifshitz transition which gives rise to a small Fermi surface (i.e., a Fermi surface with a small $E_{F}=k_{B} T_{F}$ ) with an enhanced $T_{c}$. Thus it is important to explore if there is any particular type of interaction in a model of FBMs that could also give a relatively large $T_{c} / W$ (or $T_{c} / T_{F}$ ) as seen in the unconventional superfluids and superconductors.

In this work, we consider a model of spinless fermions and hardcore bosons on a square lattice with a filling constraint of one particle per site. While earlier studies on FBMs, for example by Kuklov and Svistunov [48], have considered the same constraint of one particle per site, they considered only fermions and bosons hopping independently, and included on-site Coulomb interaction for fermions, bosons, and between fermions and bosons. Very interestingly, the authors could show that the FBM in a commensurate optical lattice showed counterflow superfluidity. Further, while the dynamics of FBMs in the Mott phase has also been discussed in the literature, long range density wave phases have been identified for fermion-fermion and boson-boson hopping in the presence of on-site boson-boson and boson-fermion Coulomb interaction [49,50]. However, to our knowledge, a composite hopping that involves an exchange of a fermion with a boson, when they occupy neighboring sites, has not been reported to date. This form of hopping distinguishes our work from earlier work on FBMs. We allow no independent hopping. Indeed, such a hopping would be forbidden in our model as it would result in either two spinless fermions or two hardcore bosons at a site. A seemingly simple realization would be a model of strongly correlated electrons on a lattice, with an excess of electrons over lattice sites (for example, one-band Hubbard model above half filling), resulting in both singly occupied and doubly occupied sites (see Fig. 1). A pair of fermions on a site could be treated as a boson [31]. When an electron hops from a doubly occupied site to a singly occupied site, a fermion and a boson are swapped. The composite hopping mechanism provides a good description of this situation.

We briefly summarize our main results. We perform a mean-field theory of our model at zero temperature. The model displays two distinct phases separated by coupled firstorder transitions at Fermi filling fraction $\rho_{F}=\rho_{c} \simeq 0.3$ : for $\rho_{F}<\rho_{c}$ the Fermi sector is insulating and the Bose sector is a normal gas, while for $\rho_{F}>\rho_{c}$ the Fermi sector is metallic and the Bose sector is a superfluid. The fermion bandwidth varies with the Fermi energy. The fermion density of states and bulk modulus exhibit cusplike singularities at $\rho_{F}=1 / 2$, where the Fermi and Bose sectors have a density-wave (DW) order coexisting with maximum metallicity and superfluidity. The bulk modulus becomes negative for $\rho_{F}>\rho_{\kappa} \simeq 0.81$. For $\rho_{F}=1 / 2$, we make an estimate of the ratio $T_{c} / W$ in our model to be about 0.12 . This agrees closely with estimated and measured values in the range of $\sim 0.03-0.2$ for most unconventional superfluids and superconductors. The $T_{c}$ of these systems vary from about $200 \mathrm{nK}$ to $260 \mathrm{~K}$, i.e., over nine orders of magnitude. We also calculate the fermion effective
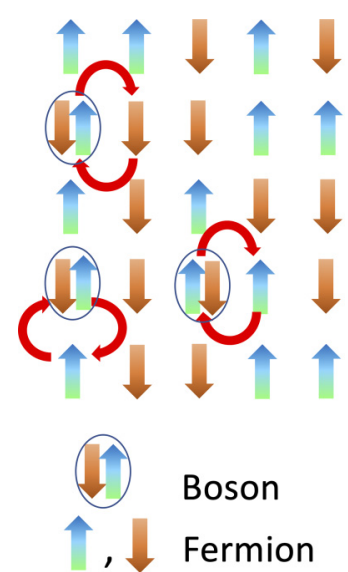

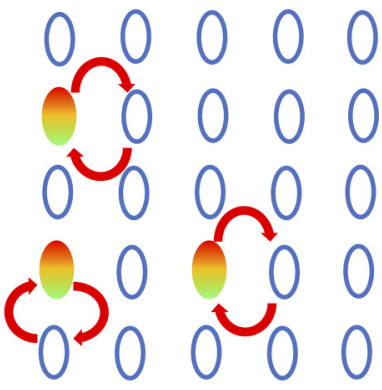

hard core Boson

spinless Fermion
FIG. 1. Schematic illustration of the composite hopping mechanism. The picture on the left shows a lattice with strongly correlated electrons, with an excess of electrons over sites, resulting in some sites being doubly occupied. An up spin on a doubly occupied site can hop to a neighboring down-spin site, leaving the down spin behind; a similar hopping is possible when the doubly occupied site neighbors an up-spin site. This hopping results in a swap of the pair and the single electron. The equivalent picture on the right results when we treat the pair as a hardcore boson and the single electron as a spinless fermion. This is the composite-hopping mechanism studied in this work.

mass $m_{e}$ that points to a divergent heavy-fermion behavior near $\rho_{F}=1 / 2$ (where it also changes sign from a particlelike to a holelike behavior) and $\rho_{F}=1$.

\section{HAMILTONIAN}

The Hamiltonian we study is

$$
\begin{aligned}
H= & -\alpha \sum_{i}\left[b_{i}^{\dagger} b_{i}+f_{i}^{\dagger} f_{i}-1\right]-\mu \sum_{i} f_{i}^{\dagger} f_{i} \\
& +\frac{U}{2} \sum_{i} b_{i}^{\dagger} b_{i}\left(b_{i}^{\dagger} b_{i}-1\right)-t \sum_{\langle i j\rangle} f_{i}^{\dagger} f_{j} b_{j}^{\dagger} b_{i},
\end{aligned}
$$

where $b_{i}^{\dagger}$ creates a spin-zero boson and $f_{i}^{\dagger}$ creates a spinless fermion at site $i$ of a two-dimensional square lattice. The parameter $\alpha$ is a Lagrange multiplier: it is used for imposing the condition that the total number of fermions and bosons be a certain given number (taken to be equal to the number of lattice sites in this work); $\mu$ is the chemical potential used to determine $\rho_{F} ; U$ is the repulsive energy between two bosons at the same site; finally, $t$ is the strength of the composite hopping: in this process a fermion hops from a site $j$ to a neighboring site $i$ when a boson located there simultaneously hops to site $j$. The boson operators satisfy $\left[b_{i}, b_{j}\right]=0=\left[b_{i}^{\dagger}, b_{j}^{\dagger}\right],\left[b_{i}, b_{j}^{\dagger}\right]=\delta_{i j}$, while the spinless-fermion operators satisfy $\left\{f_{i}, f_{j}\right\}=0,\left\{f_{i}^{\dagger}, f_{j}^{\dagger}\right\}=$ $0,\left\{f_{i}, f_{j}^{\dagger}\right\}=\delta_{i j}$, where $[a, b]=a b-b a$ and $\{a, b\}=a b+$ $b a$. In this paper, we restrict ourselves to hardcore bosons, i.e., the limit $U \rightarrow \infty$, and impose the constraint

$$
\rho_{F}+\rho_{B}=1
$$


( $\rho_{B}$ is the boson filling fraction) by requiring $\partial F / \partial \alpha=0$, with $F$ being the free energy. Given the nature of the composite hopping term, a site that is unoccupied by a fermion or a boson remains unoccupied and a site that is doubly occupied by a fermion and a boson remains doubly occupied. The only interesting case therefore is one where the constraint (2) operates, with each site being occupied either by a fermion or a boson.

\section{CALCULATION}

To calculate the properties of the Hamiltonian (1), we use the following approximation.

(1) If $\hat{F}$ is a fermion operator and $\hat{B}$ is a boson operator, then $\hat{F} \hat{B} \simeq\langle\hat{F}\rangle \hat{B}+\langle\hat{B}\rangle \hat{F}-\langle\hat{F}\rangle\langle\hat{B}\rangle$.

(2) If $\hat{B}_{i}, \hat{B}_{j}$ are boson operators at sites $i \neq j$, then $\hat{B}_{i} \hat{B}_{j} \simeq$ $\left\langle\hat{B}_{i}\right\rangle \hat{B}_{j}+\left\langle\hat{B}_{j}\right\rangle \hat{B}_{i}-\left\langle\hat{B}_{i}\right\rangle\left\langle\hat{B}_{j}\right\rangle$.

The first step implies $\langle\hat{F} \hat{B}\rangle \simeq\langle\hat{F}\rangle\langle\hat{B}\rangle$, so this is equivalent to factorizing the state space into a product of fermion and boson subspaces. The second step implies $\left\langle\hat{B}_{i} \hat{B}_{j}\right\rangle \simeq\left\langle\hat{B}_{i}\right\rangle\left\langle\hat{B}_{j}\right\rangle$, which is equivalent to further factorizing the boson subspace into a product of single-site subspaces: this is the standard mean-field decoupling used in the bosonic Hubbard model [51-57]. When we follow these steps, the composite hopping term is transformed according to

$$
\begin{aligned}
f_{i}^{\dagger} f_{j} b_{j}^{\dagger} b_{i} \simeq & \left\langle f_{i}^{\dagger} f_{j}\right\rangle\left(\left\langle b_{j}^{\dagger}\right\rangle b_{i}+\left\langle b_{i}\right\rangle b_{j}^{\dagger}-\left\langle b_{j}^{\dagger}\right\rangle\left\langle b_{i}\right\rangle\right) \\
& +\left\langle b_{j}^{\dagger}\right\rangle\left\langle b_{i}\right\rangle f_{i}^{\dagger} f_{j}-\left\langle f_{i}^{\dagger} f_{j}\right\rangle\left\langle b_{j}^{\dagger}\right\rangle\left\langle b_{i}\right\rangle,
\end{aligned}
$$

and the Hamiltonian is approximated by

$$
\begin{aligned}
H^{M F} & =H_{0}+H_{1}+H_{2}, \quad \text { where } \\
H_{0} & =N\left(2 \phi \psi^{2}+\alpha\right), \\
H_{1} & =-(\alpha+\mu) \sum_{i} f_{i}^{\dagger} f_{i}-\frac{1}{z} \psi^{2} \sum_{\langle i j\rangle} f_{i}^{\dagger} f_{j}, \\
H_{2} & =\sum_{i}\left[-\alpha b_{i}^{\dagger} b_{i}-\phi \psi\left(b_{i}+b_{i}^{\dagger}\right)\right] .
\end{aligned}
$$

We have taken $z t=1$ ( $z$ is the coordination number of the lattice), and introduced the ground-state expectation values

$$
\phi=\left\langle f_{i}^{\dagger} f_{j}\right\rangle, \quad \psi=\left\langle b_{i}\right\rangle=\left\langle b_{j}^{\dagger}\right\rangle .
$$

We assume $\phi$ and $\psi$ to be real and homogeneous. We consider $\psi$ to be the boson superfluid amplitude [51-53] and $\phi$ to be the fermion hopping amplitude. It can be observed from the expression for $H_{1}$ in Eq. (4) that when $\psi=0$, the fermion hopping term vanishes, so $\phi=0$. This indicates that when there is a superfluid transition in the Bose sector, there is an accompanying metal-insulator transition in the Fermi sector, resulting in the two transitions being always coupled. We have dropped the $U$ term: the hardcore boson limit $U \rightarrow \infty$ is incorporated by taking the single-site boson occupation number basis $\{|0\rangle,|1\rangle\}$ for diagonalizing the single-site $2 \times 2$ matrix

$$
h_{2}=\left[\begin{array}{cc}
0 & -\phi \psi \\
-\phi \psi & -\alpha
\end{array}\right]
$$

of $H_{2} / N$, with eigenvalues

$$
\lambda_{ \pm}=\frac{1}{2}[-\alpha \pm R], \quad \text { where } R=\sqrt{\alpha^{2}+4 \phi^{2} \psi^{2}} .
$$

To solve the fermion sector Hamiltonian $H_{1}$, we move over to $k$ space using the Fourier transform $f_{i}=N^{-1 / 2} \sum_{\mathbf{k}} e^{i \mathbf{k} \cdot \mathbf{r}_{\mathbf{i}}} f_{\mathbf{k}}$, so that

$$
H_{1}=\sum_{\mathbf{k}}\left(\varepsilon_{\mathbf{k}}-\mu\right) f_{\mathbf{k}}^{\dagger} f_{\mathbf{k}},
$$

where

$$
\varepsilon_{\mathbf{k}}=-\alpha-\psi^{2} \gamma_{\mathbf{k}}, \quad \gamma_{\mathbf{k}}=2\left(\cos k_{x}+\cos k_{y}\right) / z .
$$

The zero-temperature free energy per lattice site $F=H_{0} / N+$ $\left\langle H_{1}\right\rangle / N+\lambda_{-}$is now

$$
F=2 \phi \psi^{2}+\frac{1}{2}(\alpha-R)+\frac{1}{N} \sum_{\mathbf{k}}\left(\varepsilon_{\mathbf{k}}-\mu\right)\left\langle f_{\mathbf{k}}^{\dagger} f_{\mathbf{k}}\right\rangle .
$$

We observe that $(1 / N) \sum_{\mathbf{k}}\left\langle f_{\mathbf{k}}^{\dagger} f_{\mathbf{k}}\right\rangle=\rho_{F}$. Using the definition of $\phi$ in (5) and going over to $k$ space, we get

$$
\phi=\frac{1}{N z} \sum_{\langle i j\rangle}\left\langle f_{i}^{\dagger} f_{j}\right\rangle=\frac{1}{N} \sum_{\mathbf{k}} \gamma_{\mathbf{k}}\left\langle f_{\mathbf{k}}^{\dagger} f_{\mathbf{k}}\right\rangle,
$$

so that

$$
F=\frac{1}{2}(\alpha-R)+\phi \psi^{2}-(\mu+\alpha) \rho_{F} .
$$

Introducing the Fermi density of states

$$
\rho(E)=\frac{1}{N} \sum_{\mathbf{k}} \delta\left(E-\varepsilon_{\mathbf{k}}\right),
$$

we can write

$$
\phi=-\frac{1}{\psi^{2}} \int_{E_{0}}^{\mu} d E(\alpha+E) \rho(E),
$$

where $\mu$ is chosen such that the fermion filling fraction

$$
\rho_{F}=\int_{E_{0}}^{\mu} d E \rho(E)
$$

has a desired value. Here, $E_{0}=-\alpha-\psi^{2}$ is the minimum value of fermion energy. To calculate the density of states (13), we convert the $k$ sum into an integral according to $(1 / N) \sum_{\mathbf{k}} \rightarrow\left(1 / 4 \pi^{2}\right) \int d \mathbf{k}$. Since $\varepsilon_{-\mathbf{k}}=\varepsilon_{\mathbf{k}}$, the $k$-space integral is four times the integral over the first quadrant of the Brillouin zone, and so we have

$$
\rho(E)=\frac{1}{\pi^{2}} \int_{0}^{\pi} d k_{x} \int_{0}^{\pi} d k_{y} \delta\left(E+\alpha+\psi^{2} \gamma_{\mathbf{k}}\right) .
$$

The integral over $k_{y}$ can be easily evaluated, and we get

$$
\begin{aligned}
& \rho(E)=\frac{2}{\pi^{2} \psi^{2}} f\left(\frac{\alpha+E}{\psi^{2}}\right), \quad \text { where } \\
& f(u)=\int_{0}^{\pi} \frac{d k_{x}}{\sqrt{1-\left(2 u+\cos k_{x}\right)^{2}}} .
\end{aligned}
$$

We can readily see that the function $f(u)$ is real only when $-1 \leqslant u \leqslant 1$, and is non-negative. Therefore, we have the inequality $-\alpha-\psi^{2} \leqslant E \leqslant-\alpha+\psi^{2}$ for the fermion energy $E$. The Fermi bandwidth is therefore $2 \psi^{2}$. We can also see that $f(0) \rightarrow \infty$, and this is the van Hove singularity at $\rho_{F}=1 / 2$. We substitute the above expression for $\rho(E)$ into Eqs. (14) and (15) and transform the integrals to obtain

$$
\rho_{F}=\frac{2}{\pi^{2}} \int_{-1}^{u_{F}} f(u) d u, \quad \phi=-\frac{2}{\pi^{2}} \int_{-1}^{u_{F}} u f(u) d u,
$$



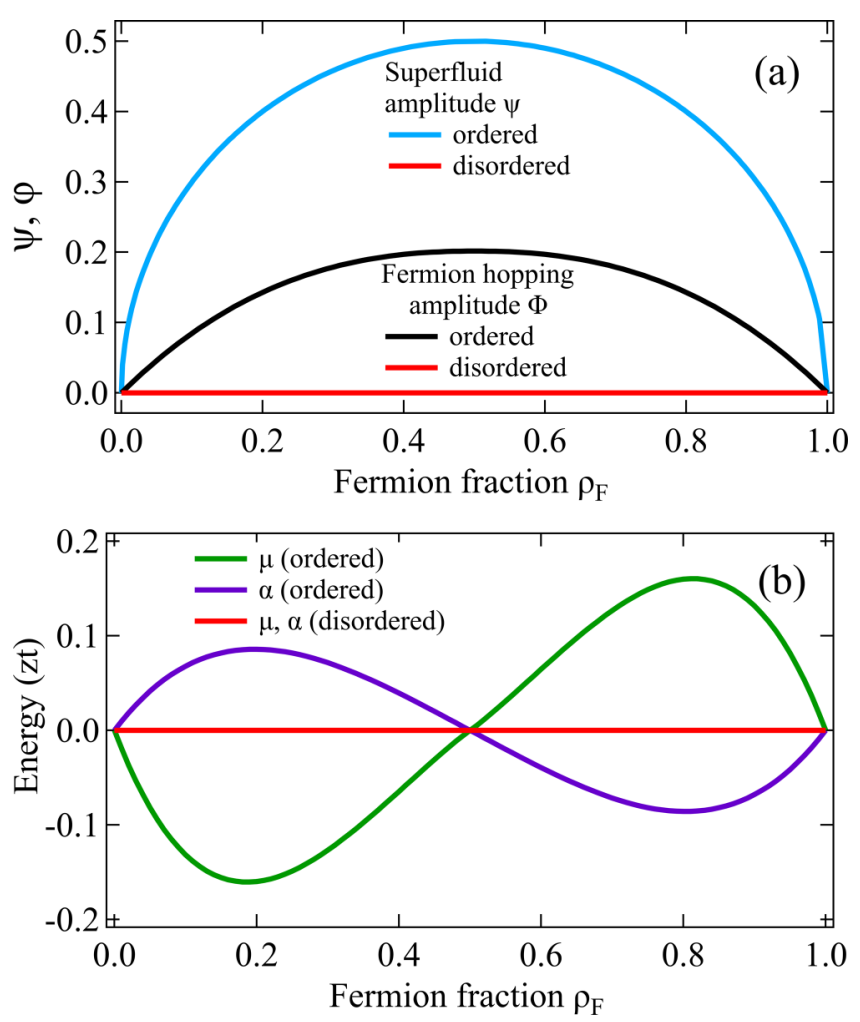

FIG. 2. (a) Plot of the order parameter $\psi$ (blue) and $\phi$ (black) as a function of $\rho_{F}$ for the ordered solution. The red line shows both in the disordered phase. (b) Plot of the chemical potential $\mu$ (green) and $\alpha$ (blue) in the ordered phase. The red line shows both in the disordered phase. The nonmonotonic behavior of $\alpha$ in the ordered phase is determined by the filling constraint [Eq. (2)], which is also responsible for a similar nonmonotonic behavior of $\mu$ in the ordered phase.

where $u_{F}=(\alpha+\mu) / \psi^{2}$. For a given $(\mu, T)$, we determine $\psi$ and $\alpha$ by simultaneously solving $\partial F / \partial \psi=2 \psi \phi(1-\phi / R)=$ 0 and $\partial F / \partial \alpha=(1 / 2)\left[\left(1-2 \rho_{F}\right)-\alpha / R\right]=0$. We obtain two solutions for $\psi$, namely $\psi=0$ (disordered, or normal Bose phase) and $R=\phi$ (ordered, or superfluid phase), and the solution $\alpha=\left(1-2 \rho_{F}\right) R$. We can write this as $\alpha^{2}[1-(1-$ $\left.\left.2 \rho_{F}\right)^{2}\right]=4 \phi^{2} \psi^{2}\left(1-2 \rho_{F}\right)^{2}$, so $\alpha=0, R=0$, and the minimum free energy $F_{0}=0$ in the disordered phase; in the ordered phase, $\alpha=\left(1-2 \rho_{F}\right) \phi, \psi^{2}=\rho_{F}\left(1-\rho_{F}\right)$, and $F_{0}=$ $\Delta$. Here $\Delta=\rho_{F}^{2}\left[-\phi+u_{F}\left(\rho_{F}-1\right)\right]$ is the minimum free energy of the ordered phase. Since $F_{0}=0$ in the disordered phase, we can interpret $\Delta$ as the energy cost of creating superfluidity. Since $u_{F}<0$ for $\rho_{F}<0.5$, it is clear that there is a certain $\rho_{c}<0.5$ such that $\Delta>0$ for $\rho_{F}<\rho_{c}$.

\section{DISCUSSION}

As mentioned above, the self-consistency equation for the order parameter $\psi$ has two solutions. For each of these solutions, we compute the quantities $\phi, \alpha$, and $\mu$. Figure 2 shows the plots of $\psi, \phi$ [panel (a)], $\alpha$, and $\mu$ [panel (b)] as functions of $\rho_{F}$.

The correct solution at each filling $\rho_{F}$ is determined based on the minimum free energy $F_{0}$. Figure 3 shows plots of $F_{0}$ in the ordered and disordered phases as functions of $\rho_{F}$.

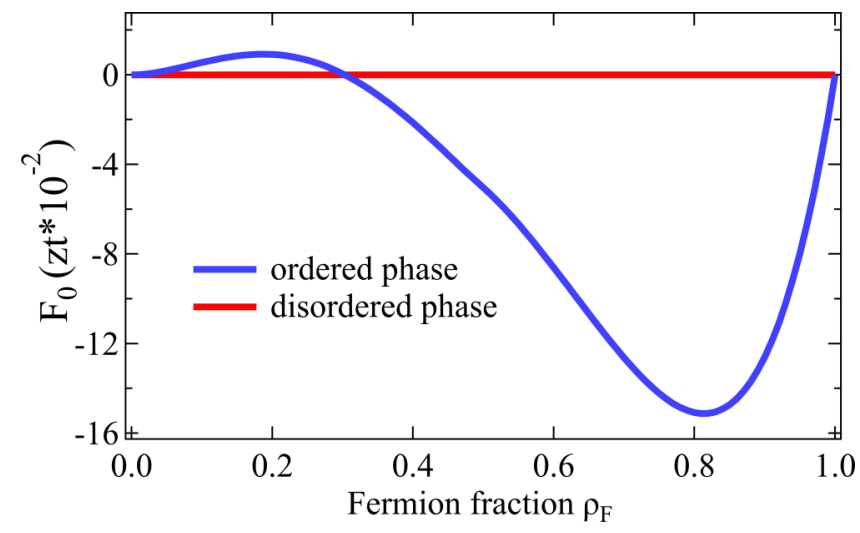

FIG. 3. Plot of the minimum free energy $F_{0}$ for the ordered (blue) and disordered (red) phases. In the ordered phase, the free energy is the cost $\Delta$ of superfluid state and becomes positive for $0<\rho_{F}<$ 0.3 , favoring the insulating normal Bose phase. At $\rho_{F}=0.3$ the free energy thus has a derivative discontinuity and we have a coupled first-order transition.

It is clear that $\Delta$, the ordered-phase free energy, is positive for $0<\rho_{F}<0.3$, indicating that the disordered phase has a lower energy than the ordered phase, and so the Bose sector does not show superfluidity for $0<\rho_{F}<0.3$. In the Bosedominated regime ( small $\rho_{F}$ ), the system therefore prefers the phase with $\psi=0$. This is unlike the bosonic Hubbard model [51-53,58], where the system is a superfluid for $0<\rho_{B}<1$ in the hardcore $(U \rightarrow \infty)$ limit.

This behavior can be understood based on model (1). In our approximation, the ground state energy per site of the model is $F_{0}=-\mu\left(\rho_{F}\right) \rho_{F}-\phi \psi^{2}$. The filling constraint $\rho_{F}+\rho_{B}=1$ removes the $\alpha$ term and makes the chemical potential filling dependent, i.e., $\mu=\mu\left(\rho_{F}\right)$. The function $\mu\left(\rho_{F}\right)$ is plotted in Fig. 2(b). It can be seen that $\mu\left(\rho_{F}\right)$ is negative for $\rho_{F}<$ $1 / 2$, and thus the superfluid ground-state energy can become positive in this regime. Numerically we find that $\Delta>0$ for $0<\rho_{F}<\rho_{c} \simeq 0.3$.

Simultaneously, for $\rho_{F}<\rho_{c}$, the Fermi sector shows an insulating phase, pointing to the important role of the coupling between Fermi and Bose sectors mediated by composite hopping. We note here that a zero-temperature insulating state with Cooper pairs has indeed been observed in amorphous bismuth films [59].

From Fig. 3 it is clear that we must pick the disordered solution for $0<\rho_{F}<0.3$ and ordered solution for $0.3<$ $\rho_{F}<1$ for each of the quantities shown in Fig. 2. When we do this, we get results with discontinuities in $\psi, \phi, \alpha, \mu$ at $\rho_{F}=0.3$ as displayed in Figs. 4(a) and 4(b). The free energy $F_{0}$ is continuous with a derivative discontinuity as shown in Fig. 5. Since there are jumps in both the Bose $(\psi)$ and Fermi $(\phi)$ sectors, we have a coupled first-order transition from an insulating normal Bose gas $(\psi, \phi=0)$ to a metallic superfluid $(\psi, \phi>0)$, as we increase $\rho_{F}$ through 0.3 .

In Fig. 6, we show a plot of the bulk modulus $\kappa=$ $\partial \mu / \partial \rho_{F}=2\left[\phi+u_{F}\left(1-2 \rho_{F}\right)\right]+\pi^{2} \psi^{2} /\left[2 f\left(u_{F}\right)\right]$. The bulk modulus has a cusplike behavior with a value of $2 \phi$ at $\rho_{F}=1 / 2$. This is because of the van Hove singularity of the function $f(u)$ which is present in the last term of the bulk modulus above. It can also be seen that $\kappa$ vanishes for $\rho_{F}=$ 

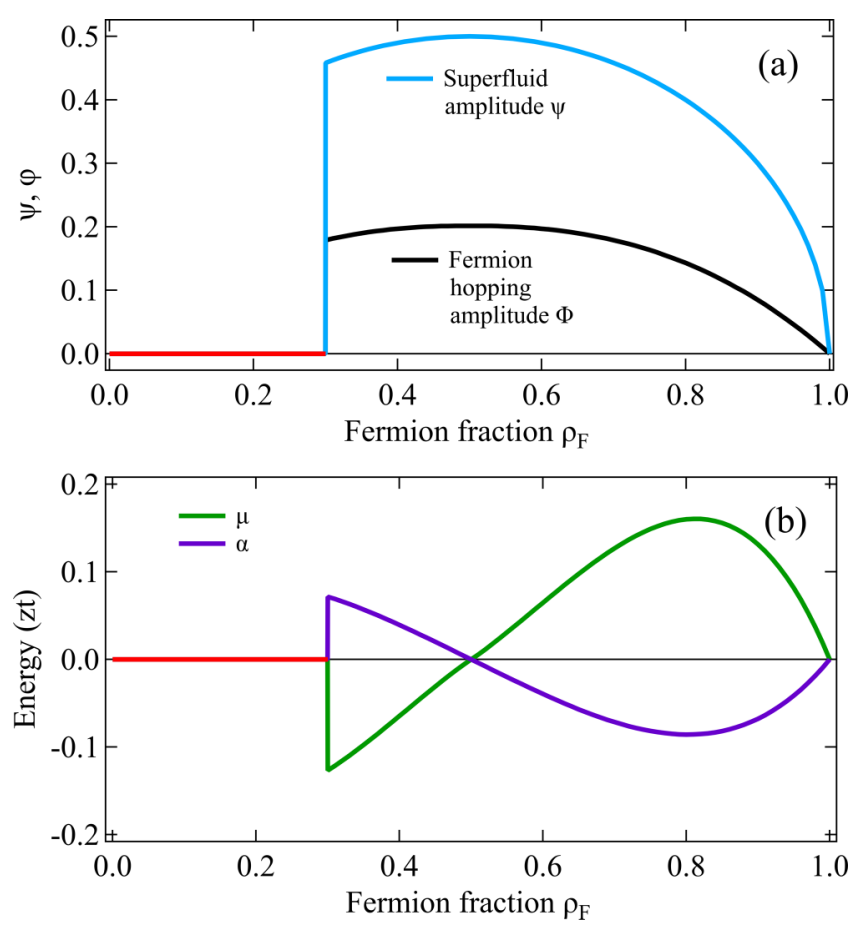

FIG. 4. Plots of (a) $\psi$ and $\phi$ and (b) $\mu$ and $\alpha$ as a function of $\rho_{F}$. At $\rho_{F} \simeq 0.3$, coupled first-order transitions are observed with jumps in $\psi, \phi, \mu$, and $\alpha$.

$\rho_{\kappa} \simeq 0.81$, becoming negative thereafter and approaching -2 at $\rho_{F}=1$. A negative value of $\kappa$ also corresponds to a negative compressibility of the fermions, which has been observed in two-dimensional electron gases [60], materials with strong spin-orbit coupling $[61,62], \mathrm{LaAlO}_{3}-\mathrm{SrTiO}_{3}$ oxide interface [63], graphene [64], and even ultracold fermionic atoms in an optical lattice [65].

We have analyzed the factors responsible for the vanishing and negative values of bulk modulus, which is directly related to the function $\mu\left(\rho_{F}\right)$ becoming decreasing for $\rho_{\kappa}<\rho_{F}<1$. First, $\mu=-\alpha+u_{F} \psi^{2}$ is determined by the filling constraint (2) that results in $\alpha\left(\rho_{F}\right)=\left(1-2 \rho_{F}\right) \phi$. Secondly, we observe that the free energy minimum $F_{0}$ is zero at $\rho_{F}=0.3$ and $\rho_{F}=$ 1 and negative in between, and is therefore bound to have

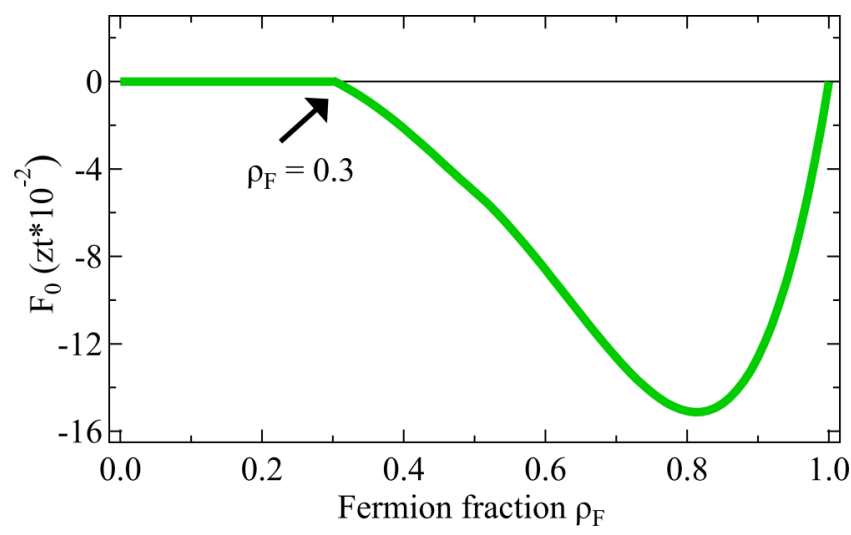

FIG. 5. Plot of the minimum free energy $F_{0}$, which is continuous with a derivative discontinuity at $\rho_{F} \simeq 0.3$.

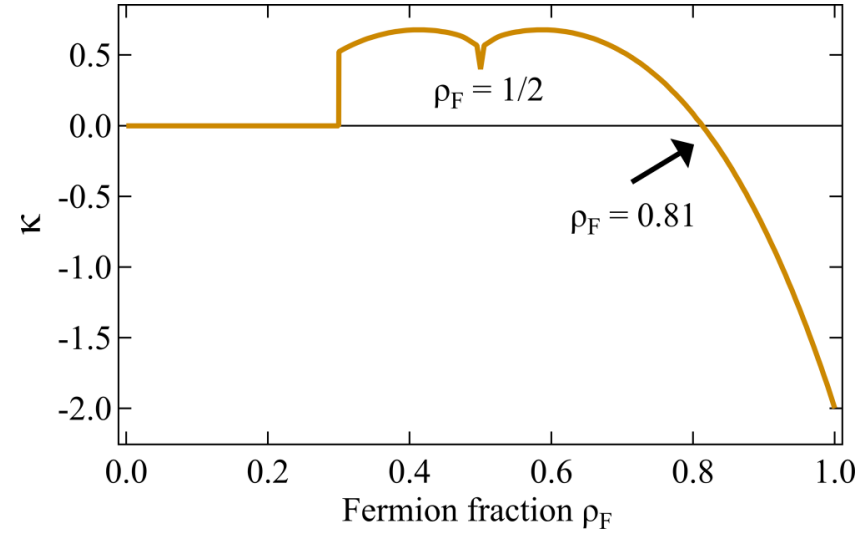

FIG. 6. Plot of the bulk modulus $\kappa$ as a function of $\rho_{F}$. For $\rho_{F}>$ $0.81, \mu$ and $F_{0}$ decrease with $\rho_{F}$, resulting in negative $\kappa$. The cusp at $\rho_{F}=1 / 2$ is a result of the van Hove singularity in the density of states.

a minimum (at $\rho_{F} \simeq 0.81$ ). This minimum is the result of a competition between the Fermi sea and the composite hopping terms, as is clear from the expression $F_{0}=-\mu\left(\rho_{F}\right) \rho_{F}-\phi \psi^{2}$ that we presented above. Since the composite hopping term $\left(-\phi \psi^{2}\right)$ is decreasing for $\rho_{F}>1 / 2$, the $\mu$ term is forced to become decreasing to the right of the $F_{0}$ minimum, with a maximum at $\rho_{F} \simeq 0.81$.

Figure 7 shows plots of Fermi band dispersion $\varepsilon_{\mathbf{k}}$ and density of states $\rho(E)$ at an arbitrary value of $\rho_{F}$. The Fermi bandwidth is $2 \psi^{2}$, completely determined by the superfluid density. Also marked is the location of the Fermi energy $\mu$ as a horizontal line. We can see that $\rho(E)$ has a cusplike singularity corresponding to energy where $\nabla_{\mathbf{k}} \varepsilon_{\mathbf{k}}$ vanishes, and this is evidently a van Hove singularity. This singularity occurs at the Fermi energy at $\rho_{F}=1 / 2$, and this is where the superfluid amplitude has a maximum, as can be seen in Fig. 2(a). A van Hove singularity near the Fermi level is an important feature of the electronic structure of

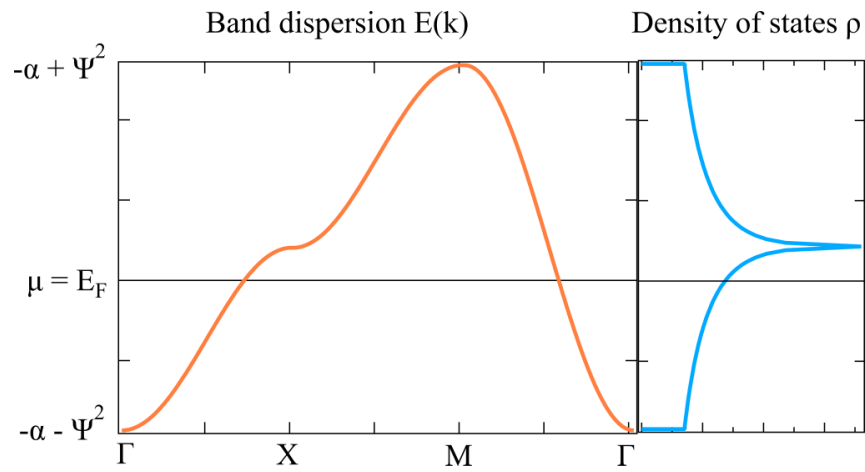

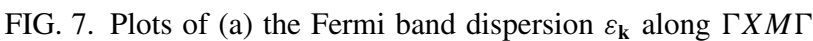
of the first Brillouin zone of the two-dimensional square lattice and (b) the Fermi density of states $\rho(E)$, at an arbitrary value of $\rho_{F}$ in the metallic phase. The two plots share a common energy axis (ordinate). Also marked is the Fermi energy $\mu$ at $\rho_{F}$ as a horizontal line between energies $-\alpha-\psi^{2}$ and $-\alpha+\psi^{2}$. It can be seen that the width of the Fermi band is $2 \psi^{2}$, and therefore varies as the Fermi level (i.e., Fermi filling fraction) changes. 

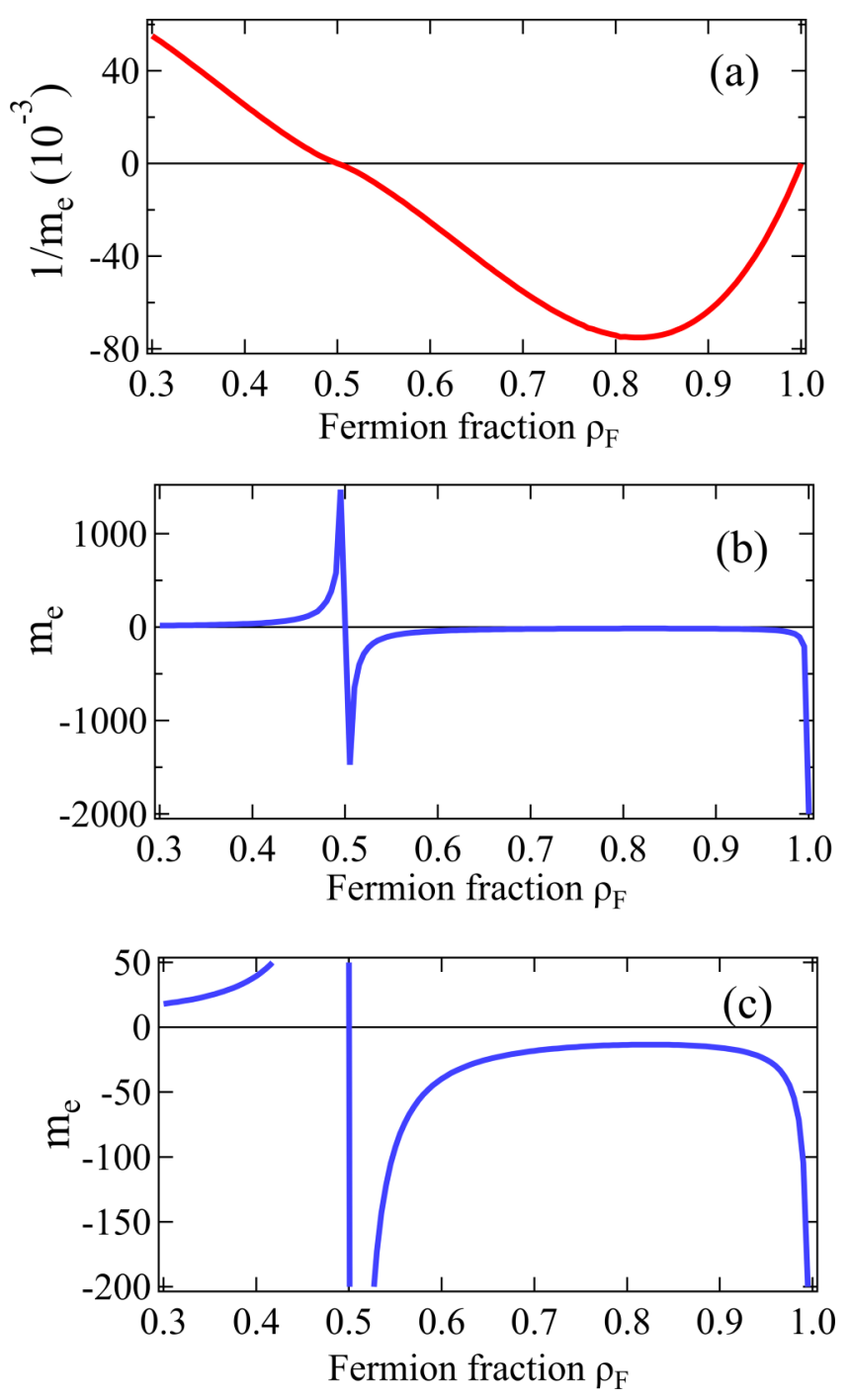

FIG. 8. Panel (a) shows the second derivative of $\varepsilon_{\mathbf{k}}$ with respect to $\mathbf{k}$ (the reciprocal of fermion effective mass $m_{e}$ ) and panels (b) and (c) show plots of $m_{e}$ with two different vertical scales to highlight its behavior near and away from the singularities at $\rho_{F}=1 / 2,1$, respectively. In (a), the minimum is at $\simeq 0.84$; this is slightly higher than where the maximum is $(\simeq 0.81)$ in the chemical potential in Fig. 1(b).

several unconventional superconductors which show a relatively high $T_{c} / T_{F}$, such as the high- $T_{c}$ cuprates [32,66-70], bilayer graphene [35,36], pressurized sulfur hydride $\mathrm{H}_{3} \mathrm{~S}$ [40,41], and lanthanum hydride $\mathrm{LaH}_{10}[44,47]$.

Figure 8(a) shows a plot of the second derivative of $\varepsilon_{\mathbf{k}}$ with respect to $\mathbf{k}$ at Fermi energy as a function of $\rho_{F}$ [see Eq. (9)] that becomes $-u_{F} \psi^{2} \equiv-(\mu+\alpha)$ upon simplification. Figures 8(b) and 8(c) show the behavior of the inverse of this second derivative, which is the Fermi effective mass $m_{e}$ on two different $y$ scales. The effective mass changes sign from particlelike $\left(\rho_{F}<1 / 2\right)$ to holelike $\left(\rho_{F}>1 / 2\right)$, and has singularities at $\rho_{F}=1 / 2$ (the van Hove point where $u_{F}=0$ ) and $\rho_{F}=1$ (where $\psi=0$ ). More interestingly, the second derivative of $\varepsilon_{\mathbf{k}}$ with respect to $\mathbf{k}$ shows a minimum at a certain $\rho_{F}$ slightly higher than $\rho_{\kappa}$, which also corresponds to a minimum of the hole effective mass. This is followed
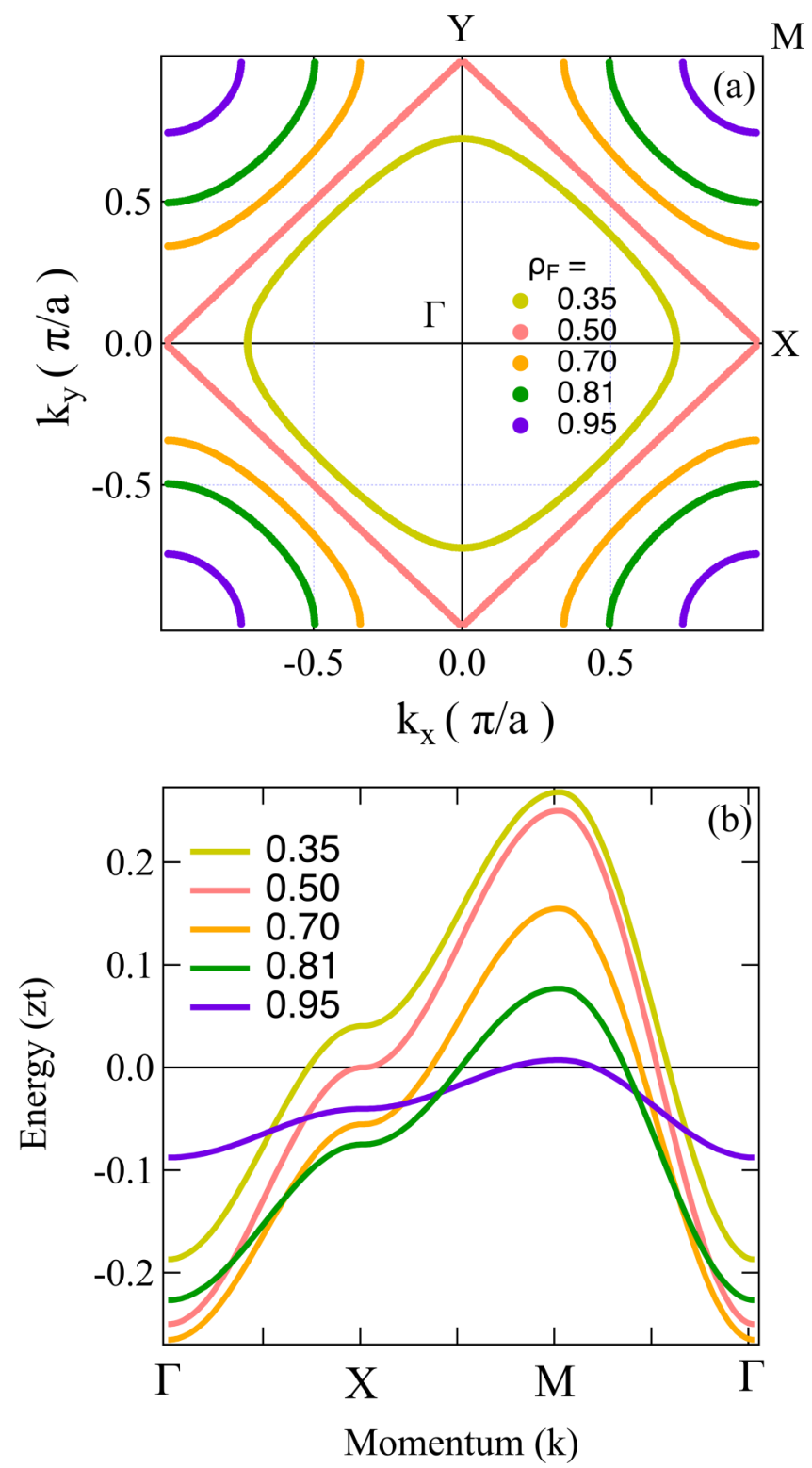

FIG. 9. (a) Plots of Fermi surface in the first Brillouin zone of the two-dimensional square lattice at five different values of $\rho_{F}$, namely $0.35,1 / 2,0.70,0.81,0.95$ in the metallic phase. It can be seen that the Fermi surface is convex (particlelike) for $\rho_{F}<1 / 2$, flat at $\rho_{F}=1 / 2$, and concave (holelike) for $\rho_{F}>1 / 2$. (b) Plot of band dispersions for $\rho_{F}=0.35,1 / 2,0.7,0.81$, and 0.95 , corresponding to the particle and hole Fermi surfaces shown in (a).

by a systematic increase in the effective mass for $\rho_{F}>\rho_{\kappa}$, culminating in a divergence at $\rho_{F}=1.0$.

The Fermi surface is determined by the equation $\varepsilon_{\mathbf{k}}=\mu$, that simplifies to $\gamma_{\mathbf{k}}=-u_{F}$. Figure 9 (a) shows plots of Fermi surface in the first Brillouin zone at five different values of $\rho_{F}$, namely $0.35,1 / 2,0.70,0.81,0.95$, in the metallic phase. It can be seen that, as a function of filling, the nature of carriers changes from particlelike for $\rho_{F}<1 / 2$ (convex surface at $\rho_{F}=0.35$ ) to holelike for $\rho_{F}>1 / 2$ (concave surfaces at $\left.\rho_{F}=0.70,0.81,0.95\right)$, with a flat surface at $\rho_{F}=1 / 2$. This behavior is qualitatively similar to that of a tight-binding model. What is unusual, and distinguishes our model from 
TABLE I. List of superfluid/superconducting systems and their characteristic parameters: transition temperature $T_{C}$, bandwidth (W)/Fermi temperature $\left(T_{F}\right)$, and ratio $T_{C} / W$ or $T_{C} / T_{F}$. The last column lists the reference numbers for the data sources.

\begin{tabular}{|c|c|c|c|c|}
\hline System & $T_{c}$ & $W / T_{F}$ & $\begin{array}{l}T_{C} / W \\
T_{C} / T_{F}\end{array}$ & References \\
\hline $\mathrm{Nb}$ & $9.3 \mathrm{~K}$ & $\sim 6.18 \times 10^{4} \mathrm{~K}$ & $1.5 \times 10^{-4}$ & {$[46]$} \\
\hline $\mathrm{Sn}$ & $3.72 \mathrm{~K}$ & $\sim 11.8 \times 10^{4} \mathrm{~K}$ & $3.15 \times 10^{-5}$ & {$[46]$} \\
\hline LAO-STO & $0.31 \mathrm{~K}$ & $\sim 230 \mathrm{~K}$ & 0.0013 & {$[71,72]$} \\
\hline High- $T_{c}$ cuprates & $\sim 153 \mathrm{~K}$ & $4600-5800 \mathrm{~K}$ & $0.026-0.033$ & [32-34] \\
\hline Bilayer graphene & $1.7 \mathrm{~K}$ & $24 \mathrm{~K}$ & 0.07 & {$[35,36]$} \\
\hline $\begin{array}{l}\text { Resonant ultracold } \\
\text { Fermi }\end{array}$ & $<240 \mathrm{nK}$ & $1.2 \mu \mathrm{K}$ & $<0.2$ & [37] \\
\hline $\begin{array}{l}\text { Ultracold Bose-Fermi } \\
\text { mixture }\end{array}$ & $260 \mathrm{nK}$ & $\sim 1.4 \mu \mathrm{K}$ & 0.19 & {$[5]$} \\
\hline Bulk $\mathrm{Fe}_{1+y}\left(\mathrm{Se}_{0.4} \mathrm{Te}_{0.6}\right)$ & $14 \mathrm{~K}$ & $116 \mathrm{~K}$ & 0.12 & {$[17]$} \\
\hline $\begin{array}{l}\text { Monolayer FeSe } \\
\text { on STO }\end{array}$ & $75 \mathrm{~K}$ & $580 \mathrm{~K}$ & 0.13 & {$[38]$} \\
\hline $\mathrm{H}_{3} \mathrm{~S}$ & $203 \mathrm{~K}$ & $1740 \mathrm{~K}$ & 0.12 & {$[39-41]$} \\
\hline $\mathrm{LaH}_{10}$ & $260 \mathrm{~K}$ & $1160 \mathrm{~K}$ & 0.22 & [42-44] \\
\hline $\begin{array}{l}\text { Composite hopping } \\
\text { model }\end{array}$ & $0.06 z t$ & $0.5 z t$ & 0.12 & This work \\
\hline
\end{tabular}

the tight-binding model, is that the fermion bandwidth $2 \psi^{2}$ changes as the chemical potential $\mu$ changes, as shown in the plots of Fig. 9(b), where we show the band dispersions for $\rho_{F}=0.35,1 / 2,0.7,0.81$, and 0.95 . It is observed that the bandwidth is maximum at $\rho_{F}=1 / 2$ (which corresponds to the case of the van Hove singularity at the Fermi energy), and decreases for values of $\rho_{F}$ below and above that. It is also clear that the band dispersions for $\rho_{F}=0.35$ is particlelike and centered at the $\Gamma$ point, while for $\rho_{F}=0.7,0.81$, and 0.95 , they are holelike and centered at the $M$ point in the Brillouin zone.

Finally, we consider the correlation function $C(\mathbf{r})=$ $(1 / N) \sum_{i}\left\langle\delta \rho_{\mathbf{r}_{i}} \delta \rho_{\mathbf{r}_{i}+\mathbf{r}}\right\rangle$, where $\delta \rho_{\mathbf{r}_{i}}=f_{i}^{\dagger} f_{i}-\rho_{F}$ is the deviation from mean fermion density at lattice site $\mathbf{r}_{i}$. Using Wick's theorem we write the expectation value of a four-fermion operator as a sum of products of expectation values of two-fermion operators. We then obtain $C(\mathbf{r})=-n^{2}(\mathbf{r})$, where $n(\mathbf{r})=(1 / N) \sum_{\mathbf{k}}\left\langle f_{\mathbf{k}}^{\dagger} f_{\mathbf{k}}\right\rangle e^{i \mathbf{k} \cdot \mathbf{r}}$, and $C_{\mathbf{q}}=-(1 / N) \sum_{\mathbf{k}}\left\langle f_{\mathbf{k}}^{\dagger} f_{\mathbf{k}}\right\rangle\left\langle f_{\mathbf{k}+\mathbf{q}}^{\dagger} f_{\mathbf{k}+\mathbf{q}}\right\rangle$ for the Fourier transform of $C(\mathbf{r})$. Figure 10 shows the plots of $n(\mathbf{r})$ and $C_{\mathbf{q}}$ for several values of $\rho_{F} . n(\mathbf{r})$ has been plotted along the $x$ axis as a function of lattice spacing. It has a periodicity of twice the lattice spacing for $\rho_{F}=1 / 2$, but no apparent periodicity for other values of $\rho_{F}$. This is also supported by the plot of $C(\mathbf{q})$ in (b) that shows a cusplike singularity for $\rho_{F}=1 / 2$ at the $M$ point with $\mathbf{q}=(\pi, \pi)$, but is nearly featureless for other values of $\rho_{F}$.

For $\rho_{F}=1 / 2$, we can analytically evaluate the $\mathbf{k}$-space integrals to obtain $\pi^{2} n(\mathbf{r})=(\cos \pi y-\cos \pi x) /\left(x^{2}-y^{2}\right)$. This shows that there is a density wave (DW) with wave vector $\mathbf{Q}=(\pi, \pi)$ : the square lattice is divided into two subsquare lattices with twice the lattice constant, with fermions occupying one of them and bosons occupying the other. In this case, every fermion has a boson as its nearest neighbor and vice versa. This enhances composite hopping and leads to maximum superfluidity and metallicity, as we saw in Fig. 2(a).

We write $\left\langle f_{\mathbf{k}}^{\dagger} f_{\mathbf{k}}\right\rangle \equiv g\left(\varepsilon_{\mathbf{k}}\right)$, where the function $g(x)$ is 1 if $x \leqslant \mu$ and zero otherwise, so that $C_{\mathbf{q}}=$ $-(1 / N) \sum_{\mathbf{k}} g\left(\varepsilon_{\mathbf{k}}\right) g\left(\varepsilon_{\mathbf{k}+\mathbf{q}}\right)$. For $\rho_{F}=1 / 2$, we have $\alpha=0$, so from Eq. (9) we have $\varepsilon_{\mathbf{k}+\mathbf{Q}}=-\varepsilon_{\mathbf{k}}$. For $\mathbf{q}=\mathbf{Q}$, we can use this nesting property of the Fermi surface and convert the $\mathbf{k}$ sum to an integral over the density of states to obtain $C_{\mathbf{Q}}=-\rho(0)(\mu-0)=0$, in agreement with Fig. 10(b). This points to the role of Fermi surface nesting in causing a DW order for $\rho_{F}=1 / 2$.

The filling $\rho_{F}=1 / 2$ turns out to be very special: we have a van Hove singularity resulting in a cusp in $\kappa$, and Fermi surface nesting resulting in a DW phase coexisting with a maximum in Bose superfluid and Fermi hopping amplitudes. In this case, $F_{0} \simeq 0.06 z t$ can be taken to be a reasonable approximation of $T_{c}$, the critical temperature for BEC. The Fermion band, as we saw in Fig. 7, has a width of $W \simeq 0.5 z t$, giving us an estimate of $T_{c} / W \simeq 0.12$. In Table I, we present values of the ratio $T_{c} / W$ (or $T_{c} / T_{F}$ ) for a variety of unconventional superfluids and superconductors [5,32-44,71,72], for which $T_{c}$ varies over about nine orders of magnitude, from $\sim 200 \mathrm{nK}$ to $260 \mathrm{~K}$. For the hydrides, $\mathrm{H}_{3} \mathrm{~S}$ and $\mathrm{LaH}_{10}$, while early reports discussed a BCS type mechanism involving high energy phonons, the validity of the Migdal approximation has been questioned recently $[40,41,47]$. It is noted that for all the systems, $T_{c}, T_{F}$, or $E_{F}$ values were obtained from experimental values reported in the literature (references listed in Table I), while for $\mathrm{H}_{3} \mathrm{~S}$ and $\mathrm{LaH}_{1} 0$, the $T_{F}$ or $E_{F}$ values were estimated from band structure calculations $[41,44]$. The ratio in most cases is between 0.03 and 0.2 , and the estimate from our model agrees very closely with these values. For comparison, we also include corresponding values for two conventional superconductors: elemental $\mathrm{Nb}$ metal and Sn metal [46]. 


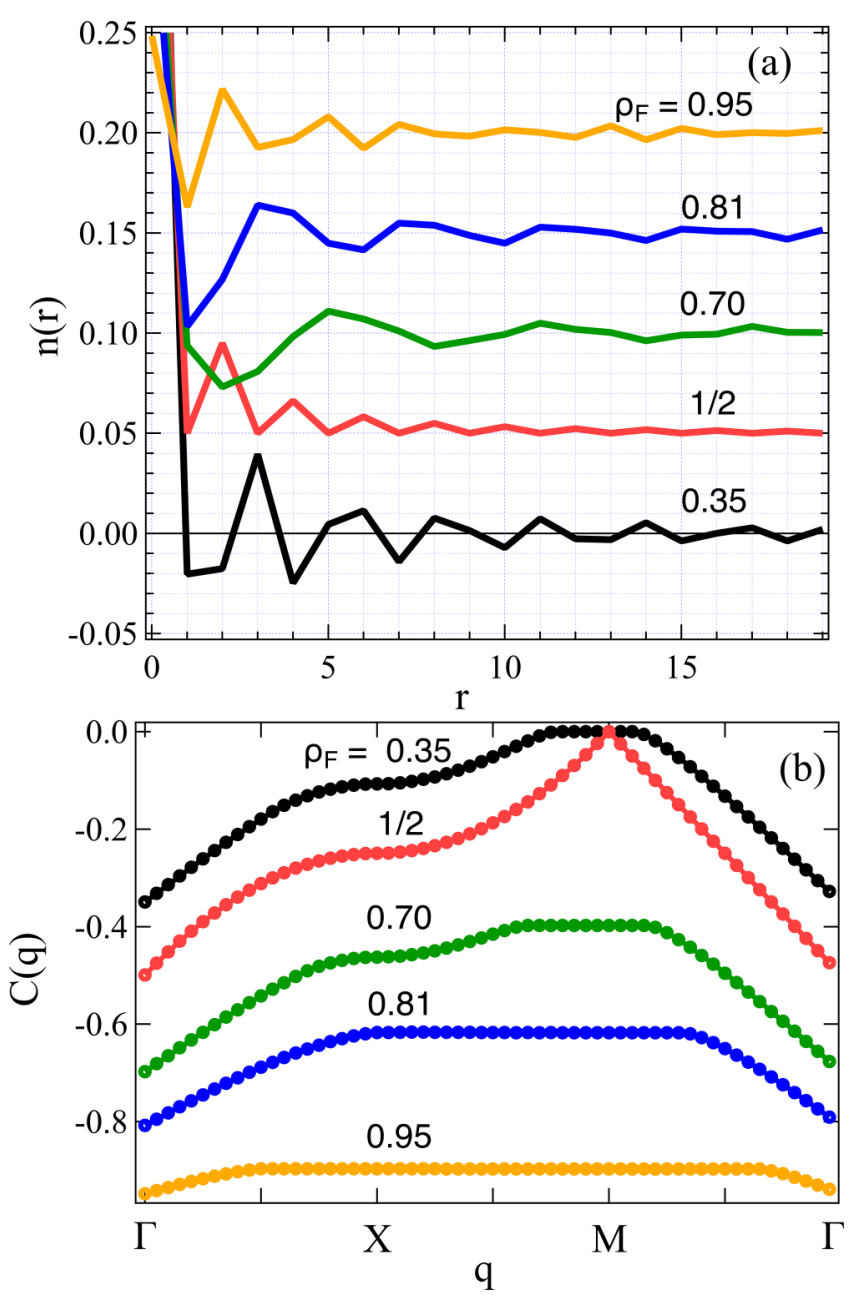

FIG. 10. (a) Plots of the function $n(\mathbf{r})$ [see text; $C(\mathbf{r})=-n^{2}(\mathbf{r})$ ] along the $x$ direction of the square lattice and (b) of the Fourier transform $C(\mathbf{q})$ along the $\Gamma X M \Gamma$ direction in $\mathbf{q}$ space, for $\rho_{F}=$ $0.35,1 / 2,0.70,0.81,0.95$ in the metallic phase. The curves in (a) are shifted along the $y$ axis for clarity. Observe the periodicity of $n(\mathbf{r})$ for $\rho_{F}=1 / 2$ (red), with a wavelength of twice the lattice spacing, indicating a density wave (DW) ordering in this case. The cusp in $C(\mathbf{q})$ at the $M$ point for $\rho_{F}=1 / 2$ (red) corresponds to the nesting vector along the $\Gamma-M$ direction.

We note a few unusual features of the insulating and metallic phases of the Fermi sector. We have insulating behavior at a range of partial fillings of the Fermi band. This is unlike the usual case where the insulating behavior is associated with a band gap. In our model, the insulating behavior of the Fermi sector results from the energy cost of the superfluid phase of the Bose sector. This results in the insulating Fermi sector for $0<\rho_{F}<0.3$. In contrast, the insulating behavior for $\rho_{F}=1$ results from a divergent effective mass $m_{e}$ due to narrowing of the fermion band (see Fig. 8). The metallic phase close to the van Hove point $\rho_{F}=1 / 2$ is also unusual because the metallicity (as measured by $\phi$ ) is maximum while $m_{e}$ has a large magnitude.

\section{CONCLUSION}

In conclusion, we have presented a model of FBMs with a hopping mechanism that exhibits several interesting properties at zero temperature. In the Bose-dominated regime, the model is in an insulating normal phase: the Fermi sector is insulating, while the Bose sector is a normal gas. As the Fermi filling fraction is increased, the model has coupled first-order transitions at $\rho_{F} \simeq 0.3$, where superfluid amplitude $\psi$ and fermion hopping amplitude $\phi$ jump to finite nonzero values. For $\rho_{F}>0.3$, the system is a metallic superfluid: the Fermi sector is metallic, while the Bose sector is a superfluid, with maximum values of $\psi, \phi$ coexisting with a DW order at $\rho_{F}=$ $1 / 2$. There is a van Hove singularity in the density of states at Fermi energy that makes the bulk modulus $\kappa$ develop a cusplike minimum for $\rho_{F}=1 / 2 ; \kappa$ vanishes at $\rho_{F} \simeq 0.81$ and is negative for $\rho_{F}>0.81$, as a result of a competition between Fermi sea and composite-hopping contributions to the groundstate energy. The fermion bandwidth varies with the chemical potential with a maximum at $\rho_{F}=1 / 2$, the van Hove point. The fermion effective mass displays singular behavior at $\rho_{F}=1 / 2,1$. And, finally, our estimate for the ratio $T_{c} / W$ agrees closely with reported ratios for a variety of unconventional superfluids and superconductors that have $T_{c}$ values spread over nine orders of magnitude between $\sim 200 \mathrm{nK}$ and $260 \mathrm{~K}$.

\section{ACKNOWLEDGMENTS}

A.C. thanks the Ministry of Science and Technology of the Republic of China, Taiwan, for financially supporting this research under Contract No. MOST 108-2112-M-213-001MY3.
[1] C. Ebner and D. O. Edwards, The low temperature thermodynamic properties of dilute solutions of ${ }^{3} \mathrm{He}$ in ${ }^{4} \mathrm{He}$, Phys. Rep. 2, 77 (1970).

[2] A. G. Truscott, K. E. Strecker, W. I. McAlexander, G. B. Patridge, and R. G. Hulet, Observation of Fermi pressure in a gas of trapped atoms, Science 291, 2570 (2001).

[3] F. Schreck, L. Khaykovich, K. L. Corwin, G. Ferrari, T. Bourdel, J. Cubizolles, and C. Salomon, Quasipure BoseEinstein Condensate Immersed in a Fermi Sea, Phys. Rev. Lett. 87, 080403 (2001).

[4] Z. Hadzibabic, C. A. Stan, K. Dieckmann, S. Gupta, M. W. Zwierlein, A. Gorlitz, and W. Ketterle, Two-Species Mixture of
Quantum Degenerate Bose and Fermi Gases, Phys. Rev. Lett. 88, 160401 (2002).

[5] I. Ferrier-Barbut, M. Delehaye, S. Laurent, A. T. Grier, M. Pierce, B. S. Rem, F. Chevy, C. Salomon, A mixture of Bose and Fermi superfluids, Science 345, 1035 (2014).

[6] M. Randeria and E. Taylor, Crossover from Bardeen-CooperSchrieffer to Bose-Einstein condensation and the unitary Fermi gas, Annu. Rev. Condens. Matter Phys. 5, 209 (2014).

[7] W. Ketterle and M. W. Zwierlein, in Proceedings of the International School of Physics: Enrico Fermi, edited by M. Inguscio, W. Ketterle, and C. Salomon (IOS Press, London, 2008), pp. 95-287. 
[8] L. V. Keldysh and A. N. Kozlov, Collective properties of excitons in semiconductors, Sov. Phys. JETP 27, 521 (1968).

[9] B. O. Kerbikov, BCS-Bose crossover in color superconductivity, Phys. At. Nucl. 65, 1918 (2002).

[10] M. Bartenstein, A. Altmeyer, S. Riedl, S. Jochim, C. Chin, J. Hecker Denschlag, and R. Grimm, Crossover from a Molecular Bose-Einstein Condensate to a Degenerate Fermi Gas, Phys. Rev. Lett. 92, 120401 (2004).

[11] C. A. Regal, C. Ticknor, J. L. Bohn, and D. S. Jin, Tuning pWave Interactions in an Ultracold Fermi Gas of Atoms, Phys. Rev. Lett. 90, 053201 (2003).

[12] T. D. Cumby, R. A. Shewmon, M.-G. Hu, J. D. Perreault, and D. S. Jin, Feshbach-molecule formation in a Bose-Fermi mixture, Phys. Rev. A 87, 012703 (2013).

[13] R. S. Bloom, M.-G. Hu, T. D. Cumby, and D. S. Jin, Tests of Universal Three-Body Physics in an Ultracold Bose-Fermi Mixture, Phys. Rev. Lett. 111, 105301 (2013).

[14] Y. Lubashevsky, E. Lahoud, K. Chashka, D. Podolsky, and A. Kanigel, Shallow pockets and very strong coupling superconductivity in $\mathrm{FeSe}_{x} \mathrm{Te}_{1-x}$, Nat. Phys. 8, 309 (2012).

[15] K. Okazaki, Y. Ito, Y. Ota, Y. Kotani, T. Shimojima, T. Kiss, S. Watanabe, C.-T. Chen, S. Niitaka, T. Hanaguri, H. Takagi, A. Chainani, and S. Shin, Superconductivity in an electron band just above the Fermi level: possible route to BCS-BEC superconductivity, Sci. Rep. 4, 4109 (2014).

[16] S. Kasahara, T. Watashige, T. Hanaguri, Y. Kohsaka, T. Yamashita, Y. Shimoyama, Y. Mizukami, R. Endo, H. Ikeda, K. Aoyama, T. Terashima, S. Uji, T. Wolf, H. von Lohneysen, T. Shibauchi, and Y. Matsuda, Field-induced superconducting phase of FeSe in the BCS-BEC cross-over, Proc. Natl. Acad. Sci. USA 111, 16311 (2014).

[17] S. Rinott, K. B. Chashka, A. Ribak, E. D. L. Rienks, A. TalebIbrahimi, P. Le Fevre, F. Bertran, M. Randeria, and A. Kanigel, Tuning across the BCS-BEC crossover in the multiband superconductor $\mathrm{Fe}_{1+y} \mathrm{Se}_{x} \mathrm{Te}_{1-x}$ : An angle-resolved photoemission study, Sci. Adv. 3, e1602372 (2017).

[18] M. Randeria, J.-M. Duan, and L.-Y. Shieh, Bound States, Cooper Pairing, and Bose Condensation in Two Dimensions, Phys. Rev. Lett. 62, 981 (1989).

[19] R. M. Quick, C. Esebbag, and M. De Llano, BCS theory tested in an exactly solvable fermion fluid, Phys. Rev. B 47, 11512 (1993).

[20] M. J. Bijlsma, B. A. Heringa, and H. T. C. Stoof, Phonon exchange in dilute Fermi-Bose mixtures: Tailoring the FermiFermi interaction, Phys. Rev. A 61, 053601 (2000).

[21] L. Viverit, C. J. Pethick, and H. Smith, Zero-temperature phase diagram of binary boson-fermion mixtures, Phys. Rev. A 61, 053605 (2000).

[22] P. Capuzzi and E. S. Hernandez, Zero-sound density oscillations in Fermi-Bose mixtures, Phys. Rev. A 64, 043607 (2001).

[23] A. P. Albus, S. A. Gardiner, F. Illuminati, and M. Wilkens, Quantum field theory of dilute homogeneous Bose-Fermi mixtures at zero temperature: General formalism and beyond meanfield corrections, Phys. Rev. A 65, 053607 (2002).

[24] M. R. Schafroth, Theory of superconductivity, Phys. Rev. 96, 1442 (1954).
[25] J. Ranninger and S. Robaszkiewicz, Superconductivity of locally paired electrons, Physica B 135, 468 (1985).

[26] J. Ranninger, J. M. Robin, and M. Eschrig, Superfluid Precursor Effects in a Model of Hybridized Bosons and Fermions, Phys. Rev. Lett. 74, 4027 (1995).

[27] R. Friedberg and T. D. Lee, Gap energy and long-range order in the boson-fermion model of superconductivity, Phys. Rev. B 40, 6745 (1989).

[28] V. B. Geshkenbein, L. B. Ioffe, and A. I. Larkin, Superconductivity in a system with preformed pairs, Phys. Rev. B 55, 3173 (1997).

[29] T. Domanski, M. M. Maska, and M. Mierzejewski, Upward curvature of the upper critical field in the boson-fermion model, Phys. Rev. B 67, 134507 (2003).

[30] Y.-I. Shin, C. H. Schunck, A. Schirotzek, and W. Ketterle, Phase diagram of a two-component Fermi gas with resonant interactions, Nature (London) 451, 689 (2008).

[31] M. M. Maska and N. Trivedi, Temperature-driven BCS-BEC crossover in a coupled boson-fermion system, arXiv:1706.04197.

[32] A. Yamamoto, N. Takeshita, C. Terakura, and Y. Tokura, High pressure effects revisited for the cuprate superconductor family with highest critical temperature, Nat. Commun. 6, 8990 (2015).

[33] N. B. Brookes, G. Ghiringhelli, O. Tjernberg, L. H. Tjeng, T. Mizokawa, T. W. Li, and A. A. Menovsky, Detection of ZhangRice Singlets Using Spin-Polarized Photoemission, Phys. Rev. Lett. 87, 237003 (2001).

[34] B. P. Xie, K. Yang, D. W. Shen, J. F. Zhao, H. W. Ou, J. Weil, S. Y. Gu, M. Arita, S. Qiao, H. Namatame, M. Taniguchi, N. Kaneko, H. Eisaki, K. D. Tsuei, C. M. Cheng, I. Vobornik, J. Fujii, G. Rossi, Z. Q. Yang, and D. L. Feng, High-Energy Scale Revival and Giant Kink in the Dispersion of a Cuprate Superconductor, Phys. Rev. Lett. 98, 147001 (2007).

[35] Y. Cao, V. Fatemi, S. Fang, K. Watanabe, T. Taniguchi, E. Kaxiras, and P. Jarillo-Herrero, Unconventional superconductivity in magic-angle graphene superlattices, Nature (London) 556, 43 (2018).

[36] D. Marchenko, D. V. Evtushinsky, E. Golias, A. Varykhalov, Th. Seyller, and O. Rader, Extremely flat band in bilayer graphene, Sci. Adv. 4, eaau0059 (2018).

[37] C. Chin, M. Bartenstein, A. Altmeyer, S. Riedl, S. Jochim, J. Hecker Denschlag, and R. Grimm, Observation of the pairing gap in a strongly interacting Fermi gas, Science 305, 1128 (2004).

[38] Q. Song, T. L. Yu, X. Lou, B. P. Xie, H. C. Xu, C. H. P. Wen, Q. Yao, S. Y. Zhang, X. T. Zhu, J. D. Guo, R. Peng, and D. L. Feng, Evidence of cooperative effect on the enhanced superconducting transition temperature at the $\mathrm{FeSe} / \mathrm{SrTiO}_{3}$ interface, Nat. Commun. 10, 758 (2019).

[39] A. P. Drozdov, M. I. Eremets, I. A. Troyan, V. Ksenofontov, and S. I. Shylin, Conventional superconductivity at 203 kelvin at high pressures in the sulfur hydride system, Nature (London) 525, 73 (2015).

[40] A. Bianconi and T. Jarlborg, Superconductivity above the lowest Earth temperature in pressurized sulfur hydride, Europhys. Lett. (Europhysics Letters) 112, 37001 (2015). 
[41] T. Jarlborg and A. Bianconi, Breakdown of the Migdal approximation at Lifshitz transitions with giant zero-point motion in the $\mathrm{H}_{3} \mathrm{~S}$ superconductor, Sci. Rep. 6, 24816 (2016).

[42] A. P. Drozdov, P. P. Kong, V. S. Minkov, S. P. Besedin, M. A. Kuzovnikov, S. Mozaffari, L. Balicas, F. F. Balakirev, D. E. Graf, V. B. Prakapenka, E. Greenberg, D. A. Knyazev, M. Tkacz, and M. I. Eremets, Superconductivity at $250 \mathrm{~K}$ in lanthanum hydride under high pressures, Nature (London) $\mathbf{5 6 9}$, 528 (2019).

[43] M. Somayazulu, M. Ahart, A. K. Mishra, Z. M. Geballe, M. Baldini, Y. Meng, V. V. Struzhkin, and R. J. Hemley, Evidence for Superconductivity above $260 \mathrm{~K}$ in Lanthanum Superhydride at Megabar Pressures, Phys. Rev. Lett. 122, 027001 (2019).

[44] H. Liu, I. I. Naumov, R. Hoffmann, N. W. Ashcroft, and R. J. Hemley, Potential high-Tc superconducting lanthanum and yttrium hydrides at high pressure, Proc. Natl. Acad. Sci. USA 114, 6990 (2017).

[45] Y. J. Uemura, Basic Similarities Among Cuprate, Bismuthate, Organic, Chevrel-Phase and Heavy-Fermion Superconductors Shown by Penetration-Depth Measurements, Phys. Rev. Lett. 66, 2665 (1991); Condensation, excitation, pairing, and superfluid density in high-Tc superconductors: the magnetic resonance mode as a roton analog and a possible spin-mediated pairing, J. Phys.: Condens. Matter 16, S4515 (2004).

[46] N. W. Ashcroft and N. D. Mermin, Solid State Physics (Saunders College Publishing, Orlando, Florida, 1975).

[47] M. Y. Kagan and A. Bianconi, Fermi-Bose mixtures and BCSBEC crossover in High-Tc superconductors, Condens. Matter 4, 51 (2019).

[48] A. B. Kuklov and B. V. Svistunov, Counterflow Superfluidity of Two-Species Ultracold Atoms in a Commensurate Optical Lattice, Phys. Rev. Lett. 90, 100401 (2003).

[49] M. Lewenstein, L. Santos, M. A. Baranov, and H. Fehrmann, Atomic Bose-Fermi Mixtures in an Optical Lattice, Phys. Rev. Lett. 92, 050401 (2004).

[50] L. Pollet, M. Troyer, K. Van Houcke, and S. M. A. Rombouts, Phase Diagram of Bose-Fermi Mixtures in One-Dimensional Optical Lattices, Phys. Rev. Lett. 96, 190402 (2006).

[51] K. Sheshadri, H. R. Krishnamurthy, R. Pandit, and T. V. Ramakrishnan, Superfluid and insulating phases in an interacting-boson model: Mean-field theory and the RPA, Europhys. Lett. 22, 257 (1993).

[52] K. Sheshadri, H. R. Krishnamurthy, R. Pandit, and T. V. Ramakrishnan, Percolation-Enhanced Localization in the Disordered Bosonic Hubbard Model, Phys. Rev. Lett. 75, 4075 (1995).

[53] M. P. A. Fisher, P. B. Weichman, G. Grinstein, and D. S. Fisher, Boson localization and the superfluid-insulator transition, Phys. Rev. B 40, 546 (1989).

[54] W. Krauth, M. Caffarel, and J.-P. Bouchaud, Gutzwiller wave function for a model of strongly interacting bosons, Phys. Rev. B 45, 3137 (1992).

[55] R. V. Pai, K. Sheshadri, and R. Pandit, Phases and transitions in the spin-1 Bose-Hubbard model: Systematics of a mean-field theory, Phys. Rev. B 77, 014503 (2008).

[56] R. V. Pai, J. M. Kurdestany, K. Sheshadri, and R. Pandit, BoseHubbard models in confining potentials: Inhomogeneous meanfield theory, Phys. Rev. B 85, 214524 (2012).
[57] T. Kimura, S. Tsuchiya, and S. Kurihara, Possibility of a FirstOrder Superfluid-Mott-Insulator Transition of Spinor Bosons in an Optical Lattice, Phys. Rev. Lett. 94, 110403 (2005).

[58] W. Krauth and N. Trivedi, Mott and superfluid transitions in a strongly interacting lattice boson system, Europhys. Lett. (Europhysics Letters) 14, 627 (1991).

[59] M. D. Stewart, Jr., A. Yin, J. M. Xu, and J. M. Valles, Jr., Superconducting pair correlations in an amorphous insulating nanohoneycomb film, Science 318, 1273 (2007).

[60] J. P. Eisenstein, L. N. Pfeiffer, and K. W. West, Negative Compressibility of Interacting Two-Dimensional Electron and Quasiparticle Gases, Phys. Rev. Lett. 68, 674 (1992).

[61] J. He, T. Hogan, T. R. Mion, H. Hafiz, Y. He, J. D. Denlinger, S.-K. Mo, C. Dhital, X. Chen, Q. Lin, Y. Zhang, M. Hashimoto, H. Pan, D. H. Lu, M. Arita, K. Shimada, R. S. Markiewicz, Z. Wang, K. Kempa, M. J. Naughton, A. Bansil, S. D. Wilson, and R.-H. He, Spectroscopic evidence for negative electronic compressibility in a quasi-three-dimensional spin-orbit correlated metal, Nat. Mater. 14, 577 (2015).

[62] J. M. Riley, W. Meevasana, L. Bawden, M. Asakawa, T. Takayama, T. Eknapakul, T. K. Kim, M. Hoesch, S.-K. Mo, H. Takagi, T. Sasagawa, M. S. Bahramy, and P. D. C. King, Negative electronic compressibility and tunable spin splitting in $\mathrm{WSe}_{2}$, Nat. Nanotechnol. 10, 1043 (2015).

[63] L. Li, C. Richter, S. Paetel, T. Kopp, J. Mannhart, and R. C. Ashoori, Very large capacitance enhancement in a twodimensional electron system, Science 332, 825 (2011).

[64] G. L. Yu, R. Jalil, B. Belle, A. S. Mayorova, P. Blake, F. Schedin, S. V. Morozovc, L. A. Ponomarenko, F. Chiappini, S. Wiedmann, U. Zeitler, M. I. Katsnelson, A. K. Geim, K. S. Novoselov, and D. C. Elias, Interaction phenomena in graphene seen through quantum capacitance, Proc. Natl. Acad. Sci. USA 110, 3282 (2013).

[65] L. Hackermuller, U. Schneider, M. Moreno-Cardoner, T. Kitagawa, T. Best, S. Will, E. Demler, E. Altman, I. Bloch, and B. Paredes, Anomalous expansion of attractively interacting fermionic atoms in an optical lattice, Science 327, 1621 (2010).

[66] D. M. Newns, H. R. Krishnamurthy, P. C. Pattnaik, C. C. Tsuei, C. C. Chi, and C. L. Kane, Van Hove scenario for cuprate superconductivity, Physica B: Condens. Matter 186-188, 801 (1993).

[67] R. S. Markiewicz, A survey of the Van Hove scenario for highTc superconductivity with special emphasis on pseudogaps and striped phases, J. Phys. Chem. Solids 58, 1179 (1997).

[68] D. S. Dessau, Z.-X. Shen, D. M. King, D. S. Marshall, L. W. Lombardo, P. H. Dickinson, A. G. Loeser, J. DiCarlo, C.-H. Park, A. Kapitulnik, and W. E. Spicer, Key features in the measured band structure of $\mathrm{Bi}_{2} \mathrm{Sr}_{2} \mathrm{CaCu}_{2} \mathrm{O}_{8+\delta}$ : Flat bands at $\mathrm{E}_{\mathrm{F}}$ and Fermi surface nesting, Phys. Rev. Lett. 71, 2781 (1993).

[69] D. M. King, Z.-X. Shen, D. S. Dessau, D. S. Marshall, C. H. Park, W. E. Spicer, J. L. Peng, Z. Y. Li, and R. L. Greene, Observation of a Saddle-Point Singularity in $\mathrm{Bi}_{2}\left(\mathrm{Sr}_{0.97} \mathrm{Pr}_{0.03}\right)_{2} \mathrm{CuO}_{6+\delta}$ and Its Implications for Normal and Superconducting State Properties, Phys. Rev. Lett. 73, 3298 (1994).

[70] K. Gofron, J. C. Campuzano, A. A. Abrikosov, M. Lindroos, A. Bansil, H. Ding, D. Koelling, and B. Dabrowski, Observation of an "Extended" Van Hove Singularity in $\mathrm{YBa}_{2} \mathrm{Cu}_{4} \mathrm{O}_{8}$ by Ultrahigh Energy Resolution Angle-Resolved Photoemission, Phys. Rev. Lett. 73, 3302 (1994). 
[71] N. Reyren, S. Thiel, A. D. Caviglia, L. Fitting Kourkoutis, G. Hammerl, C. Richter, C. W. Schneider, T. Kopp, A.-S. Rüetschi, D. Jaccard, M. Gabay, D. A. Muller, J.-M. Triscone, and J. Mannhart, Superconducting interfaces between insulating oxides, Science 317, 1196 (2007).
[72] C. Cancellieri, A. S. Mishchenko, U. Aschauer, A. Filippetti, C. Faber, O. S. Barisic, V. A. Rogalev, T. Schmitt, N. Nagaosa, and V. N. Strocov, Polaronic metal state at the $\mathrm{LaAlO}_{3} / \mathrm{SrTiO}_{3}$ interface, Nat. Commun. 7, 10386 (2016). 